etry, trigonometry, and related branches. The models of starpolyhedra, Poinsot polyhedra, and the so-called Archimedian semi-regular solids may be mentioned as desirable for more advanced mathematical considerations.

BROWN UNIVERSITY,

R. C. Archibald. Providence, R. I.

\title{
DIFFERENTIAI GEOMETRY.
}

Leçons sur les Systèmes orthogonaux et les Coordonnées curvilignes. Par Gaston Darboux. Deuxième édition, complétée. Paris, Gauthier-Villars, 1910. 8vo. i+567 pp.

ThE first 323 pages of the present volume constitute a reprint of the first edition, which was reviewed in the BuLLETIN of January, 1899, by President E. O. Lovett. It was originally Darboux's intention to include in the complete work a number of other subjects, such as the theory of quadratic differential forms. This plan was eventually abandoned, and only such subjects are discussed in the completed volume which is now before us as are more or less directly connected with orthogonal triple systems of surfaces and families of Lamé. From the point of view of the artistic unity of the book, this change of plan is only to be commended. Let us hope however that the Fates may deal kindly with the great master of differential geometry; may the fear, which he expresses in his preface, of being prevented from completing his discussion of these other matters, prove to be unfounded; and may he be permitted to add many further contributions to the science to which he has devoted his life and which already owes him so much.

For mathematics owes a great debt to Darboux. Rarely do we find such a combination of the geometer and the analyst as is present in this master mind. He makes clear, not merely by precept but by example, that it is not enough to express a problem of geometry in analytic form and then solve the differential equations. He recognizes the true task of differential geometry as an exhibition of the complete parallelism between analysis and geometry. Not until this has been accomplished does he rest content with the solution of a problem, and many of his most notable contributions have resulted from this tendency to strive for a complete 
understanding of a mathematical situation from more than one point of view.

The present volume is divided into three books. The first two of these constitute the subject matter of the first edition, and we may refer for an account of their content to the review by Lovett which has already been mentioned. The third book begins with the exposition of certain existence theorems for systems of partial differential equations, based on the method of successive approximations, which has nowadays become thoroughly familiar to most mathematicians. The systems of partial differential equations considered are all of the form

$$
\frac{\partial u_{i}}{\partial x_{h}}=f_{\imath h}\left(u_{k}, x_{k^{\prime}}\right),
$$

where the indices $i$ and $h$ may assume different values and where the right members are given functions of the unknown functions and the independent variables.

Darboux considers three cases. First, let the system be such that one and only one of the partial derivatives of each unknown function is given by an equation of form (1). Then, under certain simple continuity conditions, there exists a unique system of solutions such that $u_{i}$ reduces to an arbitrary function of all of the $x$ 's except $x_{h}$, when $x_{h}$ assumes its initial value.

Darboux's second case is that of a completely integrable system, when all of the first order partial derivatives of each unknown function are expressible in the form (1), and when the resulting integrability conditions are satisfied.

The third case, intermediate in character, is that of a system in which several, but not all, of the first order partial derivatives of each of the unknown functions are expressible in form (1).

In Chapter II, these theorems are applied to an equation of the form

$$
\frac{\partial^{2} z}{\partial x \partial y}=f\left(x, y, z, \frac{\partial z}{\partial x}, \frac{\partial z}{\partial y}\right) .
$$

The result (already familiar from a direct investigation by Picard) is this: there exists a unique solution $z$ which reduces to a given function of $x$ for $y=y_{0}$ and to a given function of $y$ for $x=x_{0}$. A second application of the results of Chapter I is concerned with a corresponding theorem for the system 


$$
\begin{aligned}
& \frac{\partial^{2} \theta}{\partial y \partial z}=f_{1}\left(x, y, z, \frac{\partial \theta}{\partial y}, \frac{\partial \theta}{\partial z}\right), \\
& \frac{\partial^{2} \theta}{\partial z \partial x}=f_{2}\left(x, y, z, \frac{\partial \theta}{\partial z}, \frac{\partial \theta}{\partial x}\right), \\
& \frac{\partial^{2} \theta}{\partial x \partial y}=f_{3}\left(x, y, z, \frac{\partial \theta}{\partial x}, \frac{\partial \theta}{\partial y}\right) .
\end{aligned}
$$

Darboux now makes use of these theorems for the solution of some interesting problems of geometry. Let $S$ and $S_{1}$ be any two surfaces, and let us establish a point-to-point correspondence between them, such that the tangent planes at any pair of corresponding points $M$ and $M_{1}$ are parallel. There will exist in general two tangents $M t$ and $M u$ of $S$, such that the tangents $M_{1} t_{1}, M_{1} u_{1}$ of $S_{1}$ which correspond to them are respectively parallel to $M t$ and $M u$. $M t$ and $M u$ will then be conjugate tangents of $S$ at $M$, while $M_{1} t_{1}$ and $M_{1} u_{1}$ are conjugate tangents of $S_{1}$ at $M_{1}$. The curves of $S$ tangent to $M t$ and $M u$, and the curves of $S_{1}$ tangent to $M_{1} t_{1}$ and $M_{1} u_{1}$, thus form two conjugate nets which are said to be parallel. Having developed these notions, Darboux formulates the following problem. Given a surface $S$ and a conjugate net traced upon $S$; to find all of the surfaces $S_{1}$ whose points correspond to $S$ in such a way that the corresponding tangent planes are parallel and that to the given conjugate net of $S$ there corresponds on $S_{1}$ a conjugate net.

The solution is very simple. Let $C$ and $D$ be the two curves of the original net which pass through $M$. Let $M_{1}$ be any point of space and let $C_{1}$ and $D_{1}$ be any two curves through $M_{1}$ whose tangents are respectively parallel to those of $C$ and $D$. There exists one and only one surface $S_{1}$ passing through $C_{1}$ and $D_{1}$ and satisfying the conditions of the problem.

The corresponding problem in three dimensions next occupies the attention of the author. If two triple systems of surfaces or, what amounts to the same thing, two systems of curvilinear coordinates of space, can be made to correspond in such a way that at all pairs of corresponding points the tangent planes of the coordinate surfaces are parallel, Darboux speaks of them as parallel systems. It is easy to see that the coordinate curves of each system will then form networks of conjugate curves on each of the coordinate surfaces. Such a triple system of surfaces is said to be a conjugate 
system and clearly, on account of Dupin's theorem, every orthogonal system is included in this class.

The existence theorems proved in Chapter I now easily lead to the following result. Let $M$ be a point of space, and let $C_{1}, C_{2}, C_{3}$ be the three coordinate curves which, in a given conjugate system, pass through $M$. Choose any other point $M^{\prime}$ of space and let $C_{1}{ }^{\prime}, C_{2}{ }^{\prime}, C_{3}{ }^{\prime}$ be any three curves through $M^{\prime}$ subject only to the condition of having their tangents respectively parallel to those of $C_{1}, C_{2}, C_{3}$. There exists one and only one conjugate triple system of surfaces parallel to the given conjugate system and having the curves $C_{1}{ }^{\prime}, C_{2}{ }^{\prime}, C_{3}{ }^{\prime}$ among its coordinate curves.

After these preparations the general conditions for the existence of a conjugate triple system of surfaces become easily intelligible. Let $\Sigma, \Sigma_{1}, \Sigma_{2}$ be three surfaces passing through the point $M$, and let $C_{i}$ be the curve of intersection of $\Sigma_{k}$ and $\Sigma_{l}$. The three surfaces are subject only to the conditions that the tangents at $M$ to $C_{k}$ and $C_{l}$ shall be conjugate tangents of $\Sigma_{i}$. Then it is possible, in an infinite number of ways, to construct on each of the surfaces $\Sigma_{\imath}$ a conjugate net containing the curves $C_{k}$ and $C_{l}$. The choice of these three conjugate nets involves the introduction of three arbitrary functions, each of two independent variables. These having been chosen, there exists one and only one conjugate triple system containing the given three surfaces $\Sigma_{i}$ as coordinate surfaces, and the curves of the three arbitrarily chosen conjugate nets on these surfaces as coordinate lines.

The theory of conjugate triple systems is skilfully reduced by Darboux to the consideration of the following systems of partial differential equations:

$$
\begin{aligned}
& \frac{\partial \beta_{i k}}{\partial \rho_{l}}=\beta_{i l} \beta_{l k} \\
& (i \neq k \neq l), \\
& \frac{\partial U_{i}}{\partial \rho_{k}}=\beta_{i k} U_{k}, \\
& \frac{\partial H_{i}}{\partial \rho_{k}}=\beta_{k i} H_{k}, \quad(i, k, l=1,2,3), \\
& \frac{\partial u}{\partial \rho_{i}}=H_{i} U_{i},
\end{aligned}
$$

where the notation may be explained as follows. Let 


$$
x=f\left(\rho_{1}, \rho_{2}, \rho_{3}\right), \quad y=g\left(\rho_{1}, \rho_{2}, \rho_{3}\right), \quad z=h\left(\rho_{1}, \rho_{2}, \rho_{3}\right)
$$

be the expressions for the cartesian coordinates of a point in terms of the conjugate triple system of curvilinear coordinates under consideration, so that the triple system is composed of the surfaces $\rho_{1}=$ const., $\rho_{2}=$ const., $\rho_{3}=$ const. Let the direction cosines of the coordinate curves be $X_{i}, Y_{i}, Z_{i}$. Suppose now that a system of six functions $\beta_{i k}$ of $\rho_{1}, \rho_{2}, \rho_{3}$ has been obtained which satisfies $(A)$. We must then find three systems of solutions of the three equations $(B)$, which may then be identified geometrically with $X_{1}, X_{2}, X_{3} ; Y_{1}, Y_{2}, Y_{3}$; $Z_{1}, Z_{2}, Z_{3}$. It remains to find $H_{1}, H_{2}, H_{3}$ by integrating $\left(B^{\prime}\right)$, and finally to obtain $x, y, z$ by quadratures from $(C)$, identifying in order $u$ with $x, y$, and $z$.

It is clear that the integration of system $(A)$ is the most serious part of this general program. Darboux shows, in Chapter III, that there exists a single partial differential equation of the sixth order for a single function $V$ whose integration completely replaces that of system $(A)$ as far as this geometric problem is concerned.

A new notion is now introduced, that of supplementary triple systems of surfaces or, as we may say, polar systems. Whenever a conjugate triple system $S$ is given, one may find an infinite number of triple systems $\Sigma$, such that the fundamental trihedrals of the two systems at corresponding points are polar to each other, that is, such that the tangents of the coordinates lines of $\Sigma$ are perpendicular to the tangent planes of the coordinate surfaces of $S$.

In Chapter IV the author returns to orthogonal triple surfaces. Since these are also conjugate systems they must satisfy the equations $(A),(B),\left(B^{\prime}\right),(C)$. But they must also satisfy the further equations

and

$$
\frac{\partial \beta_{i k}}{\partial \rho_{i}}+\frac{\partial \beta_{k i}}{\partial \rho_{k}}+\beta_{l i} \beta_{l k}=0,
$$

$$
\frac{\partial U_{i}}{\partial \rho_{i}}=-\beta_{k i} U_{k}-\beta_{l i} U_{l}
$$

which essentially express the fact that, for an orthogonal triple system, the parallel systems and the polar systems are identical. The general existence theorems applied to the 
enlarged system of partial differential equations lead to the following result.

Let there be given three surfaces (not planes or spheres), cutting each other in pairs orthogonally along a common line of curvature. There exists one and only one orthogonal triple system containing the given three surfaces. If one of the three surfaces is a plane or sphere, to obtain a correspondingly unique result, there must be given upon it an orthogonal network of curves to take the place of the lines of curvature which in these cases are indeterminate.

Chapter V is given up to a discussion of the theorems of Combescure and Ribaucour which lead to a transformation theory enabling one to deduce from a given triple system an infinite number of others, containing additional arbitrary functions.

The author now takes up the problem anew, from the general kinematic point of view which is characteristic of his theory of surfaces. In this connection he obtains the third order differential equation of Bonnet (who was the first to notice the possibility of such a reduction), and introduces a new set of variables in terms of which the fundamental differential equation assumes a very simple form. Although these new variables are complicated by imaginary quantities, they seem to be especially well adapted for further investigations.

The main body of the book closes with a detailed consideration of triple systems which admit a continuous group of transformations of Combescure.

The four notes which follow are devoted to the application of Abel's theorem to the discovery of algebraic triple systems; to the theory of the cyclides of Dupin; to orthogonal triple systems of cyclides; and to the discussion of a special class of point transformations closely connected with the subject of orthogonal triple systems.

We may say that the new portion of this great work is characterized by a generalization of great moment. Orthogonal triple systems now appear as special cases of the more general notion of conjugate triple systems. But the theory of conjugate triple systems belongs properly to projective differential geometry, more specifically to that chapter of projective differential geometry which deals with triple systems of surfaces, a chapter which has been attacked but very recently, in a remarkable Columbia dissertation, by 
G. M. Green.* There can be little doubt but that the methods of projective differential geometry will serve to throw a flood of light upon the theory of conjugate triple systems, which promises to become a most fascinating and fruitful field for further research.

The University of Chicago,

E. J. Wilczynski. September 23, 1913.

\section{DESCRIPTIVE GEOMETRY.}

Elements of Descriptive Geometry, with applications to spherical and isometric projections, shades and shadows, and perspective. By A. E. CHURCH, late professor of mathematics in the United States military academy, and G. M. BARTLETT, instructor in descriptive geometry and mechanism in the University of Michigan. New York, American Book Company, 1911. 286 pages and 143 figures.

Practical Geometry and Graphics. By D. A. Low, professor of engineering, East London College. London, Longmans, Green and Company, 1912. 448 pages and 823 figures.

Vorlesungen über darstellende Geometrie. By GuIdo HaUcK, late professor of descriptive geometry and graphical statics in the technical school at Berlin; edited by AlFred Hauck, director of the Realschule in Schönlanke. Leipzig, Teubner, 1912. Volume I. 639 pages and 641 figures.

Lehrbuch der darstellenden Geometrie für technische Hochschulen. By EMIL MüLLER, professor of mathematics at the technical school of Vienna. Second volume, first instalment. Leipzig, Teubner, 1912. 129 pages and 140 figures.

Mr. Bartlett's revision of Church's well-known Descriptive Geometry follows the original text very closely, the main departures being in mechanical make-up. The type is larger; the articles are renumbered; important theorems and new words are printed in bold-faced type; the figures are re-drawn and put in the text itself, a number of new ones being added, in particular, two hyperboloids tangent along a generator,

* G. M. Green, "Projective differential geometry of triple systems of surfaces." Dissertation Columbia University, 1913. Press of the New Era Printing Company, Lancaster, Pa. 